\title{
PATIENT EXPERIENCE: LIVING WITH CYSTINURIA DURING THE COVID-19 PANDEMIC
}

Ramandeep Chalokia, Francesca Kum, Niamh Foran, Kay Thomas, Matthew Bultitude

Urology Centre, Guy's and St. Thomas' NHS Foundation Trust

Corresponding Author: matthew.bultitude@gstt.nhs.uk

Submitted: July 18, 2020. Accepted: July 23, 2020. Published: August 21, 2020.

\section{Background and Objectives}

\section{ABSTRACT}

Cystinuria is a rare genetic cause of recurrent urolithiasis and requires multimodal treatment, and stringent follow up. We wanted to evaluate the fears and concerns of patients with cystinuria during the COVID-19 pandemic.

\section{Materials and Methods}

An online survey using Google Forms was emailed to 165 patients with cystinuria who are maintained on a prospective database and had previously consented for email correspondence.

\section{Results}

Fifty-five responses were received following a single invitation, 23 were men and 32 women with a median age of 43 (range: $18-72$ ) years. Thirty-nine (70.9\%) out of 55 were isolating during the COVID-19 pandemic although only $7(12.7 \%)$ were given specific advice to shield officially. Six $(10.9 \%)$ reported having COVID or COVID like symptoms, but none reported having a positive test. Only $9(16.4 \%)$ had been able to work as usual. In comparison, $20(36.4 \%)$ were able to work from home. Ten out of 19 patients $(52.6 \%)$ on chelating medication described difficulty in access to their medicine, either due to a shortage at the pharmacy or prescription access. Reassuringly 34 (61.8\%) patients described no issues with access to any of their cystinuria medications.

Twenty-two patients (40\%) reported experiencing pain or passing a stone during the COVID-19 pandemic, while $30(54.5 \%)$ reported no problems. Of patients who felt it applicable, 16/31 (51.6\%) were worried about attending the Emergency Department during this time. Of patients who had scheduled hospital appointments during this time, 26/44 (59.1\%) had experienced cancellations. The majority of the respondents (37 $(67.3 \%))$ did not state any difference in the ability to stay hydrated during the lockdown.

Twenty-eight (50.9\%) were more anxious about their overall health during the pandemic, and 20 (36.4\%) had expressed more concern about their kidney stones.

\section{Conclusion}

This personal fully patient-reported snapshot of the impact of the COVID-19 pandemic on a population of cystine stone formers in the United Kingdom has highlighted additional challenges for patients with a rare disease. The lockdown measures enforced have had a varying effect on patients' ability to work as normal, access medications, health anxieties, in addition to cancellations of the planned hospital appointments and Emergency Department attendance, which will impact on their future health and pose evolving challenges in delivery of their future care.

Keywords: Cystinuria, COVID-19, pandemic, urolithiasis, rare diseases

J Endolum Endourol Vol 3(3):e1-e6; August 21, 2020

This article is distributed under the terms of the Creative Commons Attribution-Non

Commercial 4.0 International License. (C) Chalokia, et al. 


\section{INTRODUCTION}

Cystinuria is a rare genetic condition arising from an autosomal recessive defect due to mutations of two genes, SLC3A1 and SLC7A9. It results in the impaired absorption of cystine and three other amino acids, namely, ornithine, arginine, and lysine from the proximal convoluted tubule resulting in a high urinary concentration. However, it is only the poor solubility of cystine which leads to deposition and calculus formation. ${ }^{1}$ It is said to account for $1 \%$ of adult and $6 \%$ of pediatric stone disease with the mean age at diagnosis in the third decade. ${ }^{2}$

With a potential 5-year stone recurrence rate as high as $73 \%$ these patients require frequent and rigorous clinical follow up necessitating clinical review and repeat imaging, preferentially being ultrasound to avoid the radiation burden of computed tomography scanning. ${ }^{2}$ It is well known that this cohort of patients undergoes recurrent surgical interventions for recurrence of stone episodes despite aggressive medical management. This issue is far more significant in patients with poor compliance with lifestyle and medical management. ${ }^{3}$ The management of patients with cystinuria remains a formidable task for both urologists and nephrologists.

The management of cystinuria patients is even more challenging when faced with a public health crisis, such as the COVID-19 pandemic. COVID-19, is a novel severe acute respiratory syndrome coronavirus (SARS-CoV-2) which first occurred in Hubei Province, China, in early December 2019. ${ }^{4}$ On March 11th 2020, the World Health Organisation declared this a pandemic and countries were offered advice to control and tackle this global public health issue. $^{5}$ The UK government announced a nationwide lockdown in response to the rising number of cases in the country on March 23rd 2020 with the imposition of quarantine measures. ${ }^{6}$ Outpatient clinics were scaled down or replaced with telephone consultation in an endeavour to reduce trans-mission of the virus in the community. The enormity of these cancelled outpatient visits can be gauged by the fact that about 10 million outpatient appointments are usually held each month in the NHS. ${ }^{7}$

One of the significant concerns arising from the pandemic was the reluctance of the public to seek medical attention for non-COVID-19 related health conditions, with the NHS reporting a reduction in Emergency Department attendances by $57 \%$ as compared to the previous year. ${ }^{8}$

To assess the impact of COVID-19, and the associated fears and concerns of patients with cystinuria, we carried out an email survey of our cohort of cystinuria patients during this pandemic.

\section{MATERIALS AND METHODS}

In 2008 we set-up a dedicated clinic to manage adult patients with cystinuria with referrals from across the United Kingdom. From a prospectively maintained database of our clinic, we sent a single email to 165 of our patients who had previously agreed to us storing and contacting them by email. We constructed a simple online survey using Google Forms, and this was emailed on May 19th, 2020. The form did not collect patient names, and all the results are self-reported on it.

\section{RESULTS}

Fifty-five responses were received, 23 were men and 32 women with a median age of 43 (range: 18-72) years. The majority of these patients were diagnosed with cystinuria before the age of 30 , and $11 \%$ were diagnosed in the first decade of life. The stone episode preceded the actual diagnosis in the majority of the patients, and the longest time to diagnose cystinuria from the first stone episode was 39 years. Five (9\%) experienced their first stone episode after the age of 40. Twelve $(21.8 \%)$ experienced stone pain at least every six months, while $43.4 \%$ experience pain every 6-24 months. Patients self-reported passing a mean of 25 stones during their lifetime (range 0-150). Of the 55 respondents; $18(32.7 \%)$ were not currently taking medication, $21(38.2 \%)$ were on urinary alkalinisation, and $19(34.5 \%)$ were taking chelating agents (tiopronin or penicillamine). All patients had undergone surgical treatments for their stones with 33(60\%), 45(81.8\%), $38(69.1 \%)$ and $25(45.5 \%)$ respectively undergoing shockwave lithotripsy (SWL), ureteroscopy, PCNL and open surgery with a majority having more than one modality of intervention.

Thirty-nine (70.9\%) out of 55 respondents were isolating during the COVID-19 pandemic although 
only $7(12.7 \%)$ were given specific advice to shield officially. Six (10.9\%) reported having COVID or COVID like symptoms, but none reported having a positive test.

The impact of COVID-19 on patients' ability to work found that only $9(16.4 \%)$ had been able to work as usual, while $20(36.4 \%)$ were able to work from home, and $15(27.3 \%)$ had been unable to work during this period. (Figure 1).

When explicitly considering those 19/55 taking Tiopronin or Penicillamine, 10/19 (52.6\%) reported difficulty in getting their medication, either due to shortage at the pharmacy or difficulty in getting the prescription. Reassuringly 34 (61.8\%) patients described no issues with access to drugs. (Figure 2).

FIG. 1 Impact of COVID-19 on patients' ability to work. Have you been able to work during this time? 55 responses

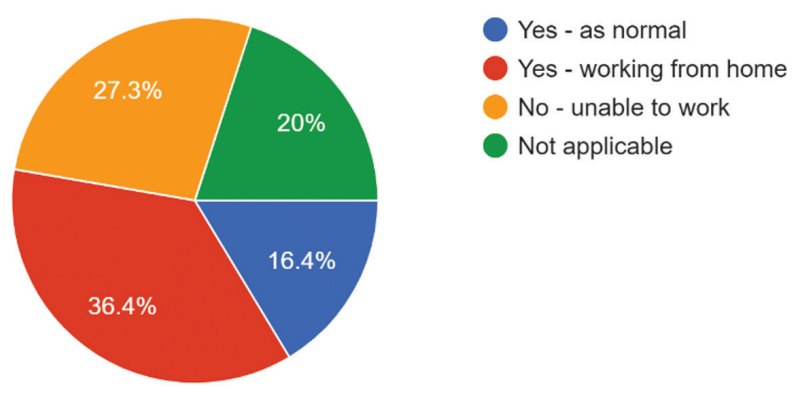

FIG. 2 Patients' experiences of difficulties in accessing medications.

Have you had difficulty obtaining your usual medications? 55 responses
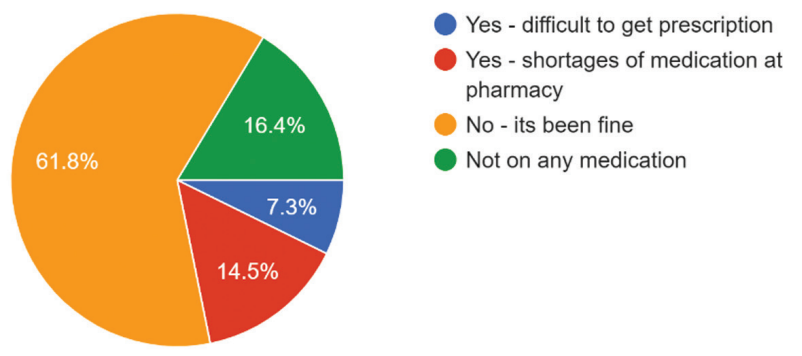

Twenty-two patients (40\%) reported experiencing pain or passing a stone during the COVID-19 pandemic, while $30(54.5 \%)$ reported no problems. Of patients who felt it applicable, 16/31 (51.6\%) were put off from attending the Emergency Department during this time by COVID-19. Of patients who had hospital appointments during this time, 26/44 (59.1\%) had experienced cancellations. Usual fluid intake for this group was a median of 3-4 litres per day, and 20 (36.4\%) wake at night to drink more fluid. (Figure 3) The majority of the respondents $(37(67.3 \%))$ did not state any difference in the ability to stay hydrated during the lockdown; however, 13 (23.6\%) had found it more manageable, and only five patients $(9.1 \%)$ had found it harder to maintain hydration which would be expected of them.

When asked about general health-related anxiety, $28(50.9 \%)$ had felt more anxious about their overall health during the pandemic, and 20 (36.4\%) had explicitly felt more concerned about their kidney stones. (Figure 4).

FIG. 3 Patient-reported fluid intake per day.

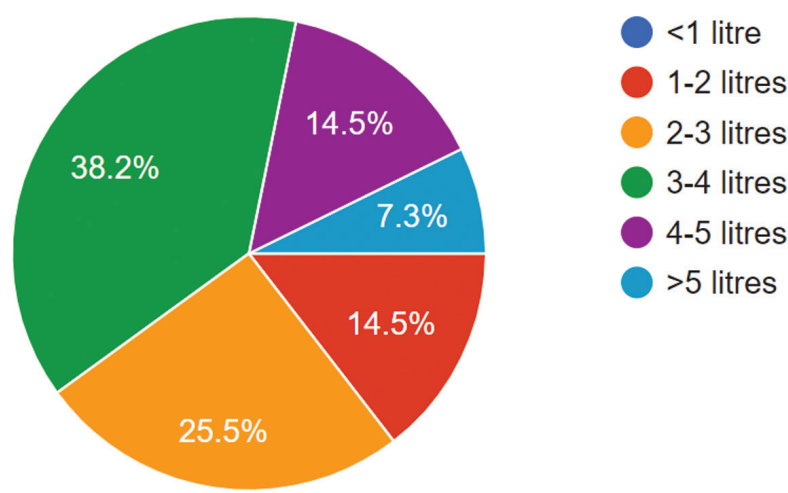

FIG. 4 (A) Patients' reported increased anxiety relating to their health during COVID-19 and (B) relating to their kidney stones.
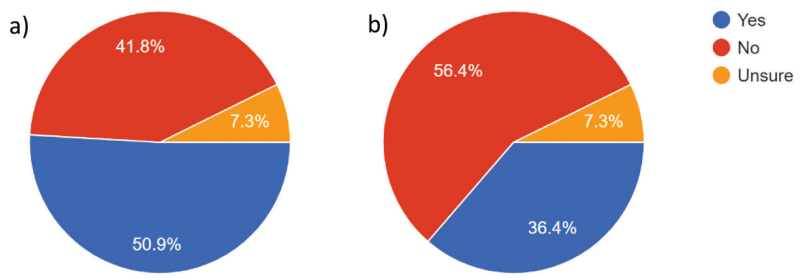

J Endolum Endourol Vol 3(3):e1-e6; August 21, 2020

This article is distributed under the terms of the Creative Commons Attribution-Non

Commercial 4.0 International License. (C) Chalokia, et al. 


\section{DISCUSSION}

This survey is first of its kind in the current literature highlighting the impact of the health crisis of the COVID-19 pandemic on a rare cohort of recurrent renal stone disease. This period has had a profound effect on patients as well as the provision of healthcare and, likely, the actual reality of what influences the pandemic has had on other conditions will not be known for some time. Our study has highlighted issues with patients isolating, unable to work, suffering increased anxiety due to health concerns, including kidney stone formation and reluctance to seek medical help if needed.

Unsurprisingly, all patients had previously undergone some intervention in their lifetime underscoring the high risk of surgical intervention in this group of metabolic stone formers. ${ }^{9}$ The proportion of patients undergoing different surgical modalities of treatment highlights the importance of having easy access to all treatments. The high numbers of open surgery ( 25 patients) is a common finding in cystinuria patients before the advent of PCNL, and many of our patients had undergone this type of surgery historically. A significant proportion had undergone ESWL (33 patients). Although cystine stones may be considered relatively resistant to SWL, it remains a common and essential form of stone treatment in cystinuria chiefly if the stone size less than $1 \mathrm{~cm} .{ }^{10}$

Prevention of stone recurrence is the goal of management in this group of patients, and therapy is directed to increase the solubility of cystine in the urine. This can be achieved in the first instance by increasing hydration resulting in hyperdiuresis, and studies have shown that urine output of $5 \mathrm{~L}$ should significantly alter the solubility of cystine and prevent stones. ${ }^{2}$ It is not pragmatic to achieve this high level of fluid intake for an extended period, and a more achiev-able objective is to have an intake of more than $3 \mathrm{~L}$ per day. ${ }^{10}$ In our survey, the majority of patients had intake varying between 2 to $4 \mathrm{~L}$, with only $4(7.3 \%)$ having an intake of more than $5 \mathrm{~L}$. It is an interesting finding of our study that $23.6 \%$ found it easier to stay hydrated at the moment and this is presumably due to isolating at home and having more time and possibly easier access to water. Very few patients reported finding it more difficult to hydrate.

Pharmacotherapy includes alkalinisation and thiolbinding drugs such as tiopronin or D-Penicillinamine. ${ }^{10}$ Using an alkalinisation agent with either potassium citrate or sodium bicarbonate raises the urine $\mathrm{pH}$ and aids in the solubility of cystine. The use of alkali is not without problems with potassium citrate often being unpalatable, with the risk of hyperkalemia which mandates regular serum potassium levels. At the same time, sodium bicarbonate causes gastrointestinal (GI) symptoms and the deleterious effects of an increased sodium load. Both require $\mathrm{pH}$ monitoring to prevent phosphate stone formation. Thiol-binding drugs are the last option to consider and act by increasing the solubility of cystine but are associated with significant side effect profiles of rash, arthralgia, leukopenia, GI intolerance, and nephrotic syndrome. These require regular monitoring of blood and urine which is problematic in a lockdown situation. Almost $60 \%$ of the patients in the survey group were currently on some form of medical therapy, either alkalisination or thiol-binding agents. Access to these medications, at least in the UK, is often difficult and even though this captured a short period of the pandemic, $7.3 \%$ of our patients had found it difficult to get their repeat prescription, and $14.5 \%$ were unable to get their medication due to shortages. The supply line for these medications is fragile at the best of times, and it is likely that if we were to survey our patients over a more extended period, then, even more, would have problems obtaining their preventative medication. It would be interesting to know how many formed stones as a result of these issues.

The majority of the respondents were isolating during the pandemic, and this was based on the government advice for those with chronic health conditions to self-isolate. ${ }^{11}$ On March 23rd the government issued guidance calling to those clinically extremely vulnerable to consider shielding and avoid face to face contact and stay at home for 12 weeks initially pending review. ${ }^{12}$ Those who were vulnerable were identified from a central database and were advised 
to shield during phase 4 of the pandemic. We did not assess co-morbidities in our survey, but the majority did not receive any advice on shielding. There was much confusion and anxiety over this as the original government advice had included those with "chronic kidney disease" (CKD) and we answered numerous requests from our cystinuria patients at this time asking for advice as to whether they had CKD and whether we thought they should shield. This high level of anxiety during the pandemic affecting the cohort is not surprising and was identified in our patients with $50.9 \%$ reporting feeling more anxious about their health and $36.4 \%$ being more worried about their kidney stones. Wang et al. studied the psychological impact and the mental health status of the pandemic on the general population. Out of that the 1201 respondents to the online survey, $54 \%$ reported the effect on their mental health as moderate to severe and almost $29 \%$ experiencing moderate to severe anxiety. ${ }^{13}$ The issue is more critical for patients with chronic diseases which require regular follow up in hospital. ${ }^{14}$

In our cohort of patients, almost half had their hospital appointments cancelled. In a prolonged lockdown situation, this could prove challenging for the patients and physicians alike as these groups require close monitoring to screen for stone recurrence, and monitor compliance, side-effects, and safety of medications. ${ }^{15}$ Alarming death figures and ${ }^{16}$ high rate of infectivity with the virus created fear and worry among the public. Many opted to avoid hospital trips even in an emergency ${ }^{8}$ as evidenced by our cohort when about $30 \%$ were afraid of seeking medical attention even when they may have experienced stone pain episodes which in normal circumstances would have prompted them to attend acute health care services.

A small proportion of our cohort of patients displayed COVID-19 like symptoms as was expected in a pandemic and unfortunately, one patient experienced complications due to COVID-19 affecting their kidneys and reported passing stones daily for one week. The fear of COVID-19 infection with associated complications is worrying enough and superimposed on having recurrent urolithiasis must have been frightening to the affected individual.

This study is not without limitations. Only a single email invitation was distributed, and although a response rate of $33 \%$ is good for this type of study, follow-up reminders may have increased the return. We cannot correlate with patient records, and therefore, the results are solely based on patient-reported responses. There may be patients who were too unwell and so unable to respond to the email; thus, we may under-report the effects of the pandemic, and no longer-term outcomes are available.

\section{CONCLUSION}

This personal fully patient-reported snapshot of the impact of the COVID-19 pandemic on a population of cystine stone formers in the United Kingdom has highlighted the additional challenges for patients with a rare disease. The COVID-19 pandemic has caused a devastating global effect on the health and well-being of many individuals. The enforced lockdown measures enforced have had a varying impact on patients' ability to work as usual, access medications, health anxieties, in addition to cancellations of the planned hospital appointments and Emergency Department attendance, which will impact on their future health and pose evolving challenges in delivery of their future care.

\section{REFERENCES}

1. Kum F, Wong K, Game D et al. Hypertension and renal impairment in patients with cystinuria: findings from a specialist cystinuria centre. Urolithiasis 2019;47(4):357-63.

2. Biyani $\mathrm{C}$ and Cartledge J. Cystinuria - diagnosis and management. EAU-EBU Update Series 2006;4(5):175-83.

3. Barbey F, Joly D, Rieu P et al. Medical treatment of cystinuria: critical reappraisal of long-term results. J Urol 2000;163(5):1419-23.

4. Wu Y, Chen C, Chan Y The outbreak of COVID-19. J Chinese Med Assoc 2020;83(3):217-220.

5. World Health Organization. WHO Director-General's opening remarks at the media briefing on COVID-19 - 11 March 2020 Available at: https://www.who.int/dg/speeches/detail/ who-director-general-s-opening-remarks-at-the-media-briefing

6. Government of the United Kingdom. Prime Minister's statement on coronavirus (COVID-19): 23 March 2020. Available at: https://www.gov.uk/government/speeches/ pm-address-to-the-nation-on-coronavirus-23-march-2020

7. National Health Service. Hospital Outpatient Activity. Available at: https://digital.nhs.uk/data-and-information/ publications/statistical/hospital-outpatient-activity 
8. The Health Foundation. How is COVID-19 changing the use of emergency care? Available at: https://www.health. org.uk/news-and-comment/charts-and-infographics/ how-is-covid-19-changing-the-use-of-emergency-care

9. Parr J, Desai D, Winkle D Natural history and quality of life in patients with cystine urolithiasis: a single centre study. BJU Internat 2015;116:31-35.

10. Thomas K, Wong K, Withington JN et al. Cystinuria-a urologist's perspective. Nat Rev Urol 2014;11(5):270-77.

11. Government of the United Kingdom. COVID-19: government announces moving out of contain phase and into delay. Available at: https://www.gov.uk/government/ news/covid-19-government-announces-moving-out-ofcontain-phase-and-into-delay

12. Department of Health and Social Care. [Internet]. Available at: https://www.england.nhs.uk/coronavirus/wp-content/ uploads/sites/52/2020/06/C0624-shielding-letter-to-nhs.pdf
13. Wang C, Pan R, Wan X et al. Immediate psychological responses and associated factors during the initial stage of the 2019 coronavirus disease (COVID-19) epidemic among the general population in China. Internat J Environ Res Public Health 2020;17(5):1729.

14. Kar SK, Yasir Arafat SM, Kabir R et al. Coping with mental health challenges during COVID-19. Coronavirus Disease 2019 (COVID-19) 2020;199-213.

15. Øbro LF, Pedersen KV, Lildal SK et al. The challenges of cystinuria in the twenty-first century: a mini review. J Rare Dis Res Treat 2016;1(3):41-45.

16. National Health Service. COVID-19 Daily Deaths. Available at: https://www.england.nhs.uk/statistics/ statistical-work-areas/covid-19-daily-deaths/ 